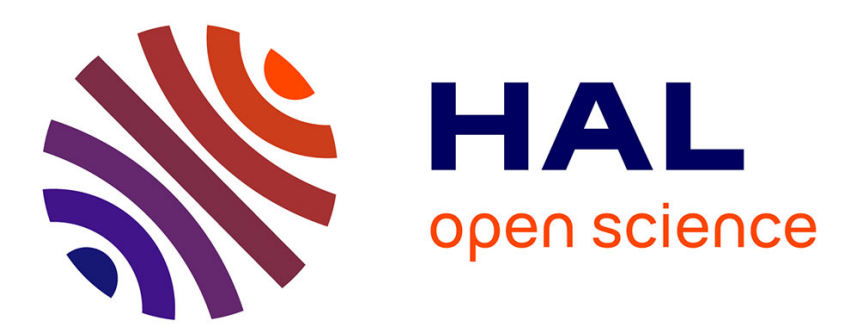

\title{
Additional SQM metrics for generic TM detection
}

\author{
Jean-Baptiste Pagot, Olivier Julien, Ikhlas Selmi
}

\section{To cite this version:}

Jean-Baptiste Pagot, Olivier Julien, Ikhlas Selmi. Additional SQM metrics for generic TM detection. ICL-GNSS 2017 International Conference on Localization and GNSS, Jun 2017, Nottingham, United Kingdom. pp.ISBN: 978-1-5386-2218-6, 10.1109/ICL-GNSS.2017.8376249 . hal-01856671

\section{HAL Id: hal-01856671 \\ https://hal-enac.archives-ouvertes.fr/hal-01856671}

Submitted on 8 Oct 2020

HAL is a multi-disciplinary open access archive for the deposit and dissemination of scientific research documents, whether they are published or not. The documents may come from teaching and research institutions in France or abroad, or from public or private research centers.
L'archive ouverte pluridisciplinaire HAL, est destinée au dépôt et à la diffusion de documents scientifiques de niveau recherche, publiés ou non, émanant des établissements d'enseignement et de recherche français ou étrangers, des laboratoires publics ou privés. 


\title{
Additional SQM metrics for Generic TM detection
}

\author{
Jean-Baptiste Pagot, Olivier Julien, Ikhlas Selmi \\ Ecole Nationale de l'Aviation Civile (ENAC) \\ Toulouse, France \\ pagot@recherche.enac.fr
}

\begin{abstract}
The ICAO Threat Model describes three cases of distortions that can occur on GPS L1 C/A signals. It defines digital and analog failures that may occur on the satellite payload. To cover a wider area of possible distortions, a Generic Threat Model (TM) based on the Fourier Transform of the signal distortions is proposed. Then, the distortion effects have to be detected and mitigated through Signal Quality Monitoring (SQM) algorithm, which is implemented on SBAS and GBAS ground infrastructures. The basic form of SQM uses simple metrics based on correlator outputs that are normalized by the correlation function prompt. Those normalized outputs can be used directly as a simple ratio metrics. They can also be combined to compute difference or sum metrics between symmetric correlator outputs pairs (with respect to the prompt) [1]. In this paper, an extended set of metrics is tested. It includes simple ratio, sum and difference between all monitored correlator outputs. The influence of several parameters related to correlator output positions is studied. The benefits provided by these new metrics compared to a baseline SQM are assessed and conclusions on the performed SQMs are made. The carried out tests are applied to Galileo E1 OS signals.
\end{abstract}

Keywords-Threat Model (TM); Generic TM; SQM; Metrics; Evil WaveForm (EWF).

\section{INTRODUCTION}

GNSS payload failure leading to a distorted transmitted signal can occur, as observed for the first time in 1993 on a GPS satellite. This distorted signal was broadcasted during a period of time without triggering any alarm to the user. Such phenomenon can create a large bias on the pseudorange measurement associated to this faulty satellite, thus creating potential hazardous situation. This is why civil aviation thoroughly investigated the potential causes and signatures of such distortions in order to characterize such event, also known as Evil WaveForm (EWF). In the early 2000's, the International Civil Aviation Organization (ICAO) standardized a Threat Model (TM) for GPS L1 C/A corresponding to this type of anomalous event. This TM was based on the knowledge of the GPS payload at the time, and on the analysis of the distortion itself. These TMs can be described as:

- TM-A is associated with failure on the navigation data unit (NDU). It can be observed on the falling edge of the positive chips as a lead or lag relative to its correct time end (digital failure).

- $\quad$ TM-B is a degradation in the analog parts that appears on the chip form as an amplitude modulation defined by the ringing frequency and a damping factor. It can be modeled as the output of a $2^{\text {nd }}$ order system with the baseband $\mathrm{C} / \mathrm{A}$ code as an input (analog failure).

- $\quad$ TM-C is combination of both TM-A and TM-B.
Besides characterizing the threat, it was also critical for civil aviation to put in place a mitigation process to protect the user from such threatening events. This led to the development of Signal Quality Monitoring (SQM) algorithms that are now present in SBAS and GBAS [2] [3]. These monitors are typically based on several correlator outputs that are used to compute detection metrics [4]. They are made to mitigate the threat characterized by the GPS L1 C/A ICAO TM distortions.

The GPS L1 C/A ICAO TM seems to be adapted to what happened to GPS L1 C/A when an EWF event occurred in the 90's [5]. However, with the use of new GNSS signals and new payloads, it is hard to say how this TM can be generalized, or can cover all possible satellite failures (until now, it seems to be well representative of GPS L1 C/A EWF threat). As a consequence, a new TM, referred to as the generic TM, has been tentatively proposed to cover extended cases of possible distortions [6]. This TM is valid for different signals and is based on a Fourier decomposition.

The proposed generic TM consists in defining the unfiltered distortion $R_{d}$ due to a payload malfunction that would affect the correlation function (from which the GNSS receiver gets synchronization) as the finite sum of cosine components according to the following model:

$$
\begin{gathered}
R_{d}(\tau)=\sum_{k=0}^{N} d_{k} \cos \left(2 \pi f_{k} \tau+\varphi_{k}\right) \\
\text { with } \sum_{k=0}^{N}\left|d_{k}\right|<D
\end{gathered}
$$

where the key parameters of the model are:

- $\quad d_{k}$ is the amplitude of the $k^{t h}$ cosine component.

- $\quad f_{k}$ is the frequency of the $k^{\text {th }}$ cosine component.

- $\quad \varphi_{k}$ is the phase of the $k^{\text {th }}$ cosine component.

- $\quad N$ is the number of components constitutive of the distortion.

- $\quad D$ is fixed to a finite value.

Obviously, this TM represents an infinite Threat Space (TS). Hence it cannot be used for testing purposes. Thus different ways have been proposed to limit the parameters space in [6]. However, a fairly wide parameters space as represented in Table 1 is deliberately kept for this study.

Finally, [6] also defined a baseline SQM to monitor the occurrence of any EWF allowed by the defined generic TM. This baseline SQM was applied to Galileo E1 OS signal and assuming a high number of available correlator outputs 
(compared to current SQM implemented in WAAS and EGNOS for instance) [1]. It was indeed anticipated that a wider EWF TM would necessarily need to be monitored by a strong SQM. The analysis showed that even with this complex SQM, the performance was not sufficient to meet the ICAO requirements on the EWF issue. The objective of this article is to go further by trying to define a more effective SQM adapted to the generic TM.

Table 1. Threat Space associated to the proposed generic TM

\begin{tabular}{|c|c|c|c|}
\hline $\boldsymbol{N}$ & $\boldsymbol{D}$ & $\boldsymbol{\varphi}_{\boldsymbol{k}}(\mathbf{r a d})$ & $\boldsymbol{f}_{\boldsymbol{k}}$ (MHz) \\
\hline$[1 ; 10]$ & 0.13 & {$[0 ; 2 \pi]$} & ] $0 ; 30]$ \\
\hline
\end{tabular}

This article is organized as follows. The first and the second parts describe SQM concepts and define a baseline SQM. They provide theoretical detection capability of the baseline SQM to detect Galileo E1 distortions within the generic TM. The third part presents the extended SQM and its new metrics. Several configurations are tested to assess the new metric benefits to detect generic TM distortions. More precisely, the influence of correlator positions on SQM performance is examined. Finally, conclusions on the presented results and the way forward will be provided.

\section{SQM PRINCIPLES}

Definitions related to SQM are provided for example in [4]. Here, only a short summary is provided.

The principle of SQM is to compute metrics (based on correlator outputs in this document) and to compare the difference between a current metric value and the metric value in the nominal case to a defined threshold.

Mathematically, the test on one metric (noted Test ${ }_{\text {metric }}^{i}$ ) is equivalent to:

$$
\text { Test }_{\text {metric }}^{i}=\frac{\text { metric }_{\text {dist }}^{i}-\text { metric }_{\text {nom }}}{\text { threshold }}
$$

where metric $_{\text {dist }}^{i}$ is the current value of the metric which can be affected by a distortion. The index $i$ shows that values are estimated based on one monitored signal $i$; metric $c_{n o m}$ is the nominal value of the metric. For example the nominal value can consist in the median of that metric across all satellites in view in nominal conditions [7].

To estimate the performance of a SQM and to know if faulty cases are detected with adequate false alarm and missed detection probabilities (resp. $P_{f f d}$ and $P_{m d}$ ), a Neyman Pearson hypothesis test is performed. The Minimum Detectable Error (MDE) provides the minimum metric bias (or distortion) that ensures that the required $P_{f f d}$ and $P_{m d}$ are met. The MDE definition based on one metric (MDE $\left.E_{\text {metric }}\right)$ and assuming a Gaussian distribution of the metric is given in [2] as:

$$
M D E_{\text {metric }}=\left(K_{m d}+K_{f f d}\right) \sigma_{m e t r i c}
$$

where $K_{f f d}=5.26$ is a typical fault-free detection multiplier representing a false detection probability of $1.5 \times 10^{-7}$ per test (ICAO requirement); $K_{m d}=3.09$ is a typical missed detection multiplier representing a missed detection probability of $10^{-3}$ per test (ICAO requirement); and $\sigma_{\text {metric }}$ is the standard deviation of the test metric at a given $C / N_{0}$.

It is assumed here that the "Gaussianity" of the metric distribution is true. Discussions about the Gaussian assumption are proposed in [4].

If several metrics are used, as it is envisaged in this paper, $P_{f f d \_m e t r i c}$ and $P_{m d_{-} m e t r i c}$ have to be computed for each individual metric. Nevertheless, as detailed in [4] even if several metrics are used to define a test, the $M D E_{\text {metric }}$ fulfilling the ICAO requirements in terms of $P_{m d}$ and $P_{f f d}$ can be modeled in a conservative way, on each metric, as:

$$
M D E_{\text {metric }}=8.35 \times \sigma_{\text {metric }}
$$

As a consequence, for each simulated distortion, a Figure Of Merit (FOM) which is the maximum value of all Test ${ }_{\text {metric }}^{i}$ considering that the threshold is equal to $M D E_{\text {metric }}$ can be computed as:

$$
\begin{aligned}
& F O M_{\text {max }}^{i} \\
& =\max _{\text {metric }}\left(\frac{\text { metric }_{\text {dist }}^{i}-\text { metric }_{\text {nom }}}{M D E_{\text {metric }}}\right)
\end{aligned}
$$

FOM $M_{\text {max }}^{i}$ represents the highest observability of the distortion by the set of metrics. It is dependent upon the monitored signal but in the following, the $i$ index will be removed as it is assumed that only one signal is looked at.

When the value of $F O M_{\max }$ exceeds 1, it means that the distortion can be detected according to the ICAO requirements.

\section{PRESENTATION OF THE BASELINE SQM}

$\overline{F O M}_{\text {max }}$ is tightly linked to the tested SQM. A baseline SQM is defined in [4] and is based on the use of 51 correlators mapping the correlation function from -0.25 to 0.25 chips with a step of 0.01 chip between correlators. Each correlator output is used to build 50 simple ratio, 25 difference ratio and 25 sum ratio metrics all normalized by the prompt correlator output. Only symmetric correlator outputs (from the prompt) are considered to build difference and sum ratio metrics used by the baseline SQM.

Fig. 1 shows theoretical baseline SQM performance regarding the generic TM applied to Galileo E1 OS signal. Tests are based on the same receiver configurations as the ones used in [6] and the tested distortions are composed of 2 sets of distortions:

- A set of 100.000 distortions (in green) that are random draws of the generic TM (10.000 distortions per number of components $\mathrm{N}$ ). This will still allow capturing the promises of the extended metrics.

- 1.000 distortions (in red) are also tested. It corresponds to a set of undetected distortions (regarding the baseline SQM) selected in [6] and that entail a worst case of differential tracking error higher than the Maximum Error Range Residual (MERR) of $1.55 \mathrm{~m}$ (according to ICAO requirements). 
One point of this figure corresponds to one simulated distortion. Simulations are done from a Matlab ${ }^{\circledR}$ software that is able to:

- Generate distorted Galileo E1C signals.

- Convolve these distorted signals with a receiver local replica to obtain distorted correlation functions.

- Assess from distorted correlation functions the tracking error and the metric test values.

In this article, the theoretical SQM performance for a given distortion is represented in the following way:

- $\quad$ The x-axis corresponds $F O M_{\max }$ values estimated at a $C / N_{0}$ equal to $30 \mathrm{~dB}-\mathrm{Hz}$. It corresponds to the theoretical "observability" of the EWF by the SQM

- The y-axis corresponds to the worst case differential tracking error over all tested airborne receiver configurations (filters, Early-Late spacings), as already described in [6].

The vertical red line represents the $F O M_{\max }$ equal to 1 and the horizontal one gives the MERR at $1.55 \mathrm{~m}$ as proposed in [6] in a L1 and L5/E5a dual frequency context. The MERR represents the error above which the tracking error becomes hazardous for the SBAS user [8].

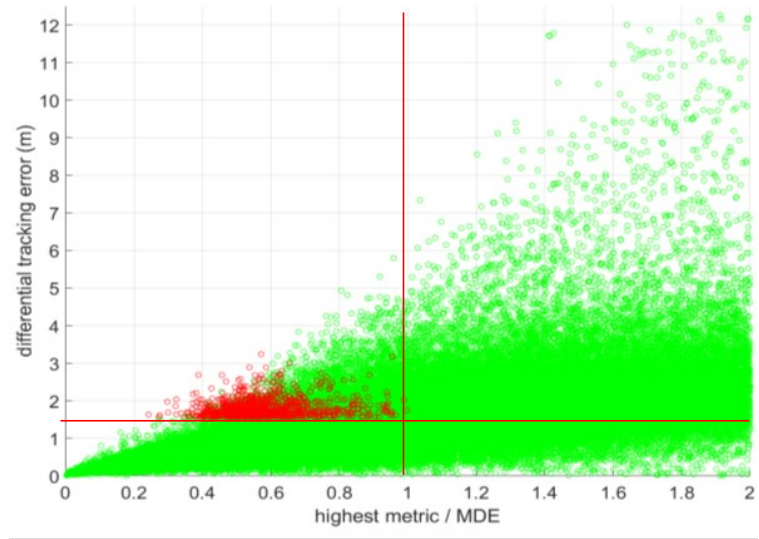

Fig. 1. Worst differential tracking error as a function of the $\mathrm{FOM}_{\max }$ at the Reference Station for Galileo E1C with the drastically reduced TS.

The threatening undetected distortions are then those appearing in the left top area limited by the two red lines. As it can be seen, the generic TM gives a great number of problematic distortions for the tested baseline SQM. The Maximum Undetectable Differential Error (MUDE) associated with these cases exceeds the MERR limit. As a consequence, the applied SQM needs to be improved in order to minimize this MUDE (ideally below the MERR).

\section{IMPROVED SQMS}

In this section new metrics are introduced and the improvement of performance that can be reached with these additional metrics compared to the three traditional metrics (simple, sum, and difference ratio metrics) are assessed.

\section{A. Aditional metrics}

The proposed additional metrics are based on the following expressions:

- the simple ratio metric is extended to (in this case, $I_{y}$ is not anymore the prompt correlator):

$$
\frac{I_{x}}{I_{y}}
$$

- the difference ratio metric (left) and the sum ratio metric (right) are extended to (in this case, $I_{x}$ and $I_{y}$ are not necessarily correlator outputs symmetric from the prompt.):

$$
\frac{I_{x}-I_{y}}{I_{0}} \quad(6) \quad \frac{I_{x}+I_{y}}{I_{0}}
$$

In the above expressions, $x$ and $y$ can take any values equal to $-0.25: 0.01: 0.25$ chip. This set of metrics will be referred to as 'extended simple metrics'. Note however that when $x$ and $y$ are equal, the corresponding metrics are useless.

Based on the above definition, $51 \times 50=2550$ metrics can be envisaged for each type of metric. Considering the symmetry aspect, it is however not necessary to test all 2550 metrics per metric type. It is sufficient to consider $x$ equal to $-0.25: 0.01: 0.25$ chip and $y$ equal to $x: 0.01: 0.25$. In these conditions, the number of metrics that is envisaged is:

- 2550 simple ratio metrics,

- 1275 difference ratio metrics,

- 1275 sum ratio metrics.

To compare in a better way the benefit of each kind of metric, extended simple ratio metrics will be divided in two categories containing 1275 metrics each:

- $\quad$ extended simple ratio metrics with $x<y$,

- $\quad$ extended simple ratio metrics with $y<x$.

\section{B. Benefit of including new metrics in the SQM}

Six metric configurations are introduced and are tested for the two sets of distortions:

- Case 1 (100 metrics): this represents the performance of the baseline SQM introduced in the previous section based on the original 100 simple ratio, simple difference and simple sum metrics.

- Case 2 (1350 metrics): the baseline SQM is tested together with the extended simple ratio metrics where $x<y$.

- Case 3 (1350 metrics): the baseline SQM is tested together with extended simple ratio metrics where $y<$ $x$. For the sake of simplicity, extended simple ratio metrics obtained when $y<x$ and noted $I_{x} / I_{y}$ are now noted $I_{y} / I_{x}$ with $x<y$.

- Case 4 (1350 metrics): the baseline SQM is tested together with extended difference ratio metrics.

- Case 5 (1350 metrics): the baseline SQM is tested together with extended sum ratio metrics. 
- Case 6 (5100 metrics): the baseline SQM is tested together with all extended ratio metrics.

Plots on Fig. 2 represent theoretical SQM performance in Case 1 (equivalent to Fig. 1) and Case 6. For the two cases of Fig. 2, the MUDE is provided. MUDE for Case 2, Case 3, Case 4 and Case 5 were estimated equal to $3.5 \mathrm{~m}, 3.6 \mathrm{~m}, 3.4 \mathrm{~m}$ and $4.1 \mathrm{~m}$ respectively. It appears that it is possible to significantly improve SQM performance using additional metrics on top of the baseline metrics. Indeed, it can be seen that the maximum differential error significantly decreases for Case 6 compared to Case 1 for the same distortion.
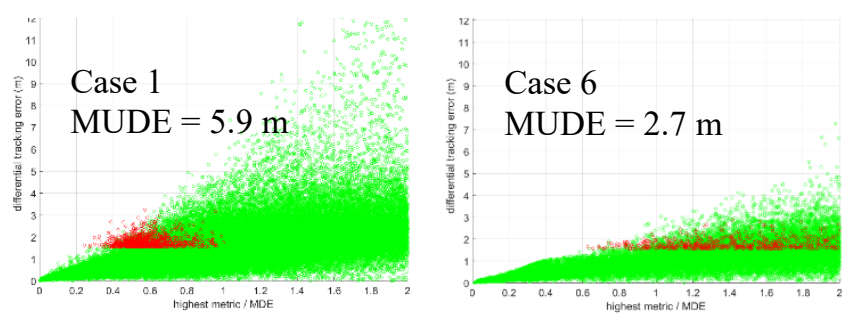

Fig. 2. Monitoring of 100000 random distortions (in green) plus the set of 1000 distortions (in red) with different SQMs based on the extended metrics.

However, these results do not allow to determine which metrics are the most useful. Sections $C$ and $D$ give primary results to understand what are the most useful metrics among the additional ones.

\section{Number of distortions with the highest impact on metrics}

This section aims at showing which metrics "detect" the highest number of distortions among the ones tested.

In the Fig. 3, one point corresponds to one metric. The x-axis corresponds to the location of $I_{x}$ with respect to the prompt. The $y$-axis corresponds to the distance between $I_{x}$ and $I_{y}$. The case 6 is represented. The color gives the number of distortions (among the 100.000) that leads to the highest test value for the different metrics considering that all extended metrics are used by the SQM. It permits to see which kind of metric is more sensitive to distortions. Yellow corresponds to a high number of detections. One plot corresponds to one kind of extended metric. One important remark is that these plots only give information about the number of distortions that are more "visible" to a given metric. In this section, the value of the differential error entailed by a distortion is not looked at.

From plots shown on Fig. 3, several conclusions are noteworthy:

- Colors can only be present below the diagonal. This result is logical because $y$ is limited to values equal to $x: 0.01: 0.25$ chip.

- The area inside the parallelogram (in red on the plot on the top left-corner) represents metrics built from one correlator output on the right of the correlation function prompt and one correlator output on the left of the correlation function prompt. It can be seen that only few distortions are well visible for these metrics.

- No metric built from correlator outputs far from each other (distance between $x$ and $y$ higher than 0.15 chip) leads to the highest value of test. Few exceptions are obtained with extended sum ratio metrics.

- Most of the distortions (82\%) affect strongly the extended sum ratio metrics, in particular those built from correlator outputs close to the prompt.
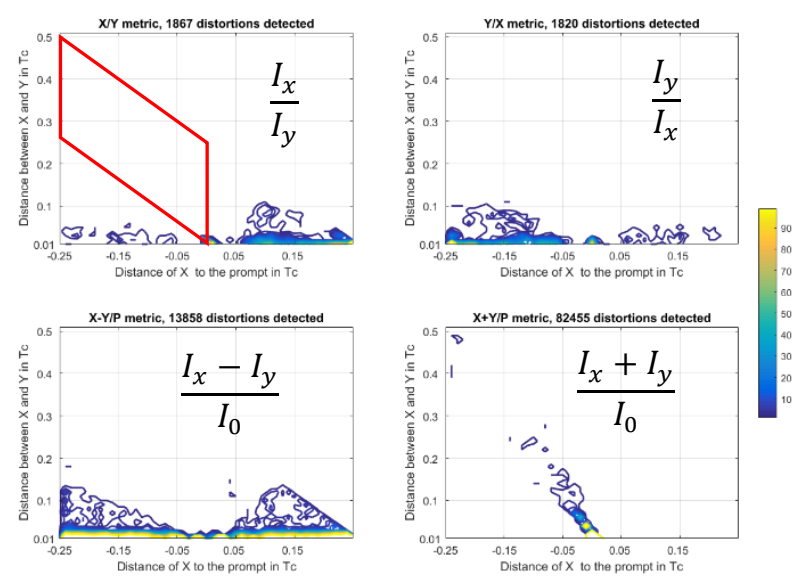

Fig. 3. Number of distortions leading to the highest test value for the different metrics, considering the 100000 random distortions and a SQM based on all extended metrics (case 6).

The most likely interpretation of the obtained results is proposed based on the mathematical expressions of the metrics standard deviations that are detailed in [4] and reminded here (for sum (difference) ratio metrics all \pm are $+(-)$ ):

$$
\begin{aligned}
& \sigma\left(\frac{I_{x} \pm I_{y}}{I_{z}}\right) \\
& =\sqrt{\frac{1}{\mu_{z}^{2}}\left[\begin{array}{c}
\left(\mu_{x} \pm \mu_{y}\right)^{2} \frac{\sigma^{2}\left(n_{z}\right)}{\mu_{z}^{2}} \\
+\sigma^{2}\left(n_{x}\right)+\sigma^{2}\left(n_{y}\right) \pm 2 \operatorname{cov}\left(n_{y} n_{x}\right) \\
-2\left(\mu_{x} \pm \mu_{y}\right) \frac{\operatorname{cov}\left(n_{x} n_{z}\right) \pm \operatorname{cov}\left(n_{y} n_{z}\right)}{\mu_{z}}
\end{array}\right]}
\end{aligned}
$$

The fact that difference ratio metrics are more able to detect distortions when the distance between $x$ and $y$ is small can be justified by the fact that the standard deviation of that metric is lower in these conditions. Indeed, the use of close correlator outputs entails an increase of $\operatorname{cov}\left(n_{y} n_{x}\right)$.

The fact that tests based on sum ratio metrics are more sensitive to distortions than other metrics (especially when correlator outputs are close to the prompt) can be justified looking at (8). Indeed, even if an increase of $\operatorname{cov}\left(n_{y} n_{x}\right)$ is entailed using two correlator outputs close to each other, an increase of the two terms $\operatorname{cov}\left(n_{x} n_{z}\right)$ and $\operatorname{cov}\left(n_{y} n_{z}\right)$ entails a decrease of the standard deviation.

By consequence, a smaller standard deviation for difference ratio metrics (resp. sum ratio metrics) using correlator outputs close to each other (resp. close to the prompt) is obtained and metrics are more sensitive to distortions.

Simple interpretations are exposed above and, for example, the impact of the term $\left(\mu_{x}-\mu_{y}\right)^{2}$ or $\left(\mu_{x}+\mu_{y}\right)^{2}$ could also be discussed to have a more precise understanding about the different metrics. 


\section{Influence of correlator outputs positioning}

1) Impact of the distance between two correlator outputs

In this part, three configurations are compared:

- Configuration 1 based on 51 correlator outputs with a distance to the prompt equal to $-0.25: 0.01: 0.25 \mathrm{chip}$. It corresponds to the location of the correlator outputs used by the baseline SQM until now. The baseline SQM then relies on 100 metrics. All extended ratio metrics (1225 metrics) presented earlier are then tested on top of the baseline SQM.

- Configuration 2 based on 25 correlator outputs with a distance to the prompt equal to $-0.24: 0.02: 0.24$ chip. The baseline SQM then relies on 48 metrics (instead of $100)$ in this configuration. All extended ratio metrics (300 metrics) are then tested on top of the baseline SQM.

- Configuration 3 based on 17 correlator outputs with a distance to the prompt equal to $-0.24: 0.03: 0.24$ chip. The baseline SQM relies on 32 metrics in this configuration and all extended ratio metrics (136 metrics) are then tested on top of the baseline SQM.

Case 1, Case 2, Case 3, Case 4, Case 5 and Case 6 (all described in the previous section) are considered even if the design of the SQM is different depending on the configuration (less correlator outputs are used). To make the comparison easier, only the upper bound of the differential tracking error is given in the following figures (rather than the cloud of points).

Results obtained for the configuration 1 are plotted on Fig. 4 and for configuration 3 in Fig. 5. From the two figures, it is noticeable that the SQM performance goes down when the number of metrics decreases. It also shows that each type of metric brings its own improvement with respect to the baseline SQM, which is the poorest of all the SQM.

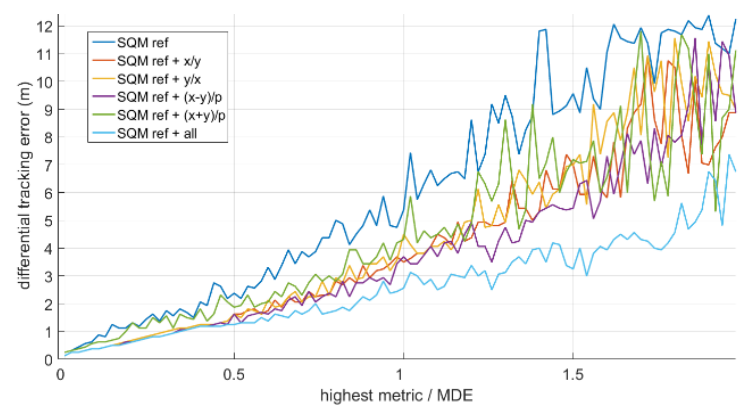

Fig. 4. Worst differential tracking error function of $\mathrm{FOM}_{\max }$ in configuration1

Interpretations can also be done from Table 2 which shows the MUDE of each tested SQM for the different configurations. This table shows that the difference between Configuration 1 and Configuration 2 is not generally significant. However, Configuration 3 brings a more significant degradation, typically of 1 meter on the MUDE. It thus seems that using correlators separated by 0.02 chip could allow a simplification of the SQM without loss of performance.

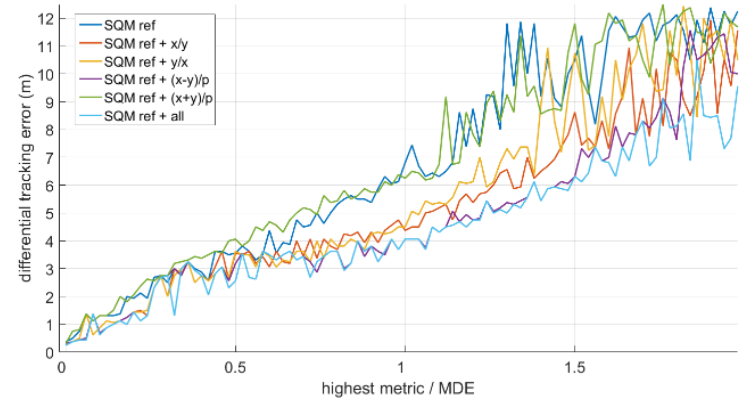

Fig. 5. Worst differential tracking error function of $\mathrm{FOM}_{\max }$ in configuration3

2) Impact of the monitored area size

In this part, three configurations are compared:

- Configuration 1 corresponds to the Configuration 1 of the previous section.

- Configuration 4 based on 25 correlator outputs with a distance to the prompt equal to $-0.12: 0.01: 0.12$ chip. The SQM relies on 48 metrics (baseline SQM) plus all extended ratio metrics (300 possible cases).

- Configuration 5 based on 13 correlator outputs with a distance to the prompt equal to $-0.06: 0.01: 0.06$ chip. The SQM relies on 24 metrics (baseline SQM) plus all extended ratio metrics (78 possible cases).

Results obtained for the configuration 1 are plotted in the previous part. Configuration 4 and configuration 5 figures are not shown in this article but were estimated. From these figures, it can be deduced that the SQM performance decreases when the monitored area decreases. This is visible on Table 2 which shows the MUDE of each SQM for the different configurations. In this case, it can be seen that the size decrease of the monitoring area has a strong impact on the MUDE. This is different from what has been observed in [4] for the ICAO-like TM. However, this is in line with the generic TM distortion observation in [6] showing that the highest values of metrics are not necessarily obtained from correlator outputs close to the main peak of the correlation function.

Table 2. MUDE in different configurations considering different metrics.

\begin{tabular}{|c|c|c|c|c|c|}
\hline & \multicolumn{5}{|c|}{ Configuration } \\
\cline { 2 - 6 } & $\mathbf{1}$ & $\mathbf{2}$ & $\mathbf{3}$ & $\mathbf{4}$ & $\mathbf{5}$ \\
\hline Case 1 & $5.9 \mathrm{~m}$ & $5.9 \mathrm{~m}$ & $6.8 \mathrm{~m}$ & $5.9 \mathrm{~m}$ & $12.1 \mathrm{~m}$ \\
\hline Case 2 & $3.5 \mathrm{~m}$ & $3.7 \mathrm{~m}$ & $4.8 \mathrm{~m}$ & $4.9 \mathrm{~m}$ & $4.9 \mathrm{~m}$ \\
\hline Case 3 & $3.6 \mathrm{~m}$ & $3.7 \mathrm{~m}$ & $4.5 \mathrm{~m}$ & $4.9 \mathrm{~m}$ & $4.9 \mathrm{~m}$ \\
\hline Case 4 & $3.4 \mathrm{~m}$ & $3.5 \mathrm{~m}$ & $4.1 \mathrm{~m}$ & $4.9 \mathrm{~m}$ & $4.9 \mathrm{~m}$ \\
\hline Case 5 & $4.1 \mathrm{~m}$ & $5.9 \mathrm{~m}$ & $6.3 \mathrm{~m}$ & $5.9 \mathrm{~m}$ & $12.1 \mathrm{~m}$ \\
\hline Case 6 & $2.7 \mathrm{~m}$ & $3.3 \mathrm{~m}$ & $4.1 \mathrm{~m}$ & $4.3 \mathrm{~m}$ & $4.9 \mathrm{~m}$ \\
\hline
\end{tabular}

Another result is put forward with Table 2: when the size of the monitored area becomes small, the extended sum ratio metrics do not improve the reference SQM capability. This result is not inconsistent with the fact that the extended sum ratio metrics are more sensitive to distortions when built from correlator outputs close to the prompt. Indeed, the most likely interpretation is that, even if a lot of distortions are detected by the sum ratio metrics built from correlator outputs close to the 
prompt, these distortions are not necessarily the most threatening for differential users (as discussed in section IV.C).

\section{3) Reducing Smartly the Number of Simple Metrics}

Section IV.D)1) showed that increasing the distance between correlators to 0.02 chip would not degrade significantly the SQM performance. Moreover, section IV.C showed that the extended simple metrics were the most effective when correlator outputs used in the metrics were as close as possible. It is thus of interest to test an SQM that is based on Configuration 2 (correlators spaced every 0.02 chip) and with extended metrics based only on immediate neighbors. Reducing the number of metrics is important to decrease probabilities of false alarm and the complexity of the processing. Fig. 6 shows the performance of such SQM based on 25 correlator outputs and 96 metrics: it brings a significant improvement, leading to a MUDE slightly above $3 \mathrm{~m}$.

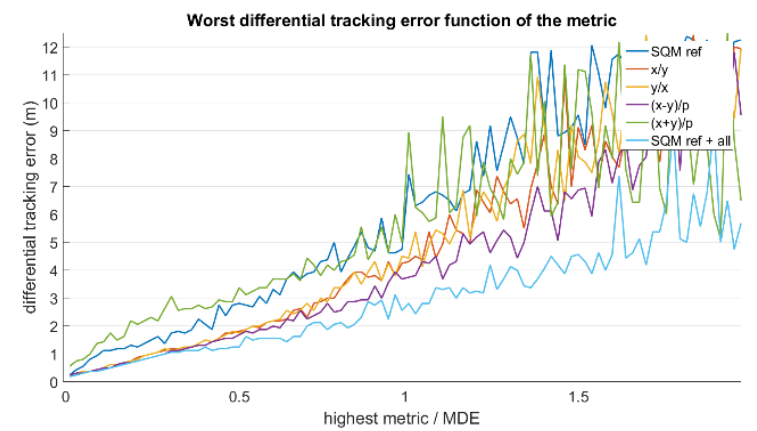

Fig. 6. Worst differential tracking error function of $\mathrm{FOM}_{\max }$ in configuration 2 considering metrics based on two consecutive correlator outputs.

Reducing the SQM to metrics that rely on two consecutive correlator outputs, does not change significantly the SQM performance (compared to the use of all available metrics) excepted for the sum ratio metric. It means that it is not necessary to use metrics built from correlator outputs too far away from each other.

\section{E. Conclusions about the addition of other simple metrics}

In this section, it was seen that the addition of new metrics can improve the performance of the SQM against the generic TM. Assuming that 51 correlator outputs are available, the MUDE was equal to $5.9 \mathrm{~m}$ with the baseline SQM and was going down to $2.7 \mathrm{~m}$ with the SQM based on all extended metrics (5100 metrics). This is a significant improvement. However this is at the expense of a very complex SQM that might not be easily manageable by the reference station.

This improvement is mainly due to the fact that more metrics are available but it can also be seen that some metrics are more sensitive to distortions than other. In this regards, the separation between correlators could be relaxed to about 0.02 chip without loss of performance due to the maximum allowed bandwidth of the reference RF front-end filter that does not let very high frequency components pass.

These promising results that would have to be further investigated, especially by testing more distortions. However, this improvement is still not sufficient to fully protect the airborne user as the MUDE is higher than the targeted MERR of $1.55 \mathrm{~m}$ even for the best SQM performance.

It has also been shown that SQM performance was sensitive to the monitored area. It was clear that the size decrease of the monitored area had a negative input with regards to the MUDE.

\section{CONCLUSIONS AND PERSPECTIVES}

In order to improve the SQM performance, many cases of simple metrics are explored. It was seen that an interesting improvement of the SQM performance was based on the use of sum and difference ratio metrics based on neighboring correlator outputs. It was also seen that the use of correlator outputs separated by 0.02 chip was a good compromise between the simplification of the SQM and the SQM performance.

Finally, it is important to mention that none of the investigated metrics were able to create a MUDE below the required MERR even if the use of additional metrics highly improves SQM performance. Another metric based on, the Alpha metric concept [7], which is a linear combination of simple ratios, could be applied to generic TM in order to improve the global MUDE.

It also seems important to work on the improvement of the SBAS SQM architecture to reach better performance, for instance by considering a centralized architecture [6].

\section{ACKNOWLEDGMENTS}

The authors gratefully acknowledge ESA for funding the work related to this paper. The opinions discussed here are those of the authors and do not necessarily represent those of ESA or other affiliated agencies.

\section{REFERENCES}

[1] R. E. Phelts, "Multicorrelator techniques for robust mitigation of threats to GPS signal quality," Stanford University, California, 2001.

[2] ICAO, "ICAO Convention - Annex 10: Aeronautical Telecommunications - Volume 1: Radio Navigation Aids," 2006

[3] R. E. Phelts, T. Walter, P. Enge, and G. Wong, "Signal deformation monitoring for dual- frequency WAAS," in Proceedings of the ION ITM, San Diego, California, 2013, pp. 93-106.

[4] J.-B. Pagot, O. Julien, P. Thevenon, F. Amarillo-Fernández, and D. Maillard, "SQM for new GNSS signals," in Proceedings of the ION GNSS+, Portland, Oregon, 2016.

[5] A. M. Mitelman, "Signal quality monitoring for GPS augmentation systems," Stanford University, California, 2004.

[6] O. Julien, I. Selmi, J.-B. Pagot, J. Samson, and F. AmarilloFernandez, "Extension of EWF threat model and associated SQM," in Proceedings of the ION ITM, Monterey, California, 2017.

[7] R. E. Phelts, T. Walter, and P. Enge, "Toward real-time SQM for WAAS: improved detection techniques," in Proceedings of the ION GNSS, Portland, Oregon, 2003, pp. 2739-27.

[8] J. Rife and R. E. Phelts, "Formulation of a time-varying maximum allowable error for Ground-Based Augmentation Systems," IEEE Transactions on Aerospace and Electronic Systems, vol. 44, no. 2, pp. 548-560, Apr. 2008. 